University of Nebraska - Lincoln

DigitalCommons@University of Nebraska - Lincoln

Robert Streubel Papers

Research Papers in Physics and Astronomy

$10-25-2021$

\title{
Ferromagnetic resonances in single-crystal yttrium iron garnet nanofilms fabricated by metal-organic decomposition
}

\author{
Szu Fan Wang \\ Kayetan Chorazewicz \\ University of California, Berkeley \\ Suvechhya Lamichhane \\ University of Nebraska-Lincoln \\ Ronald A. Parrott \\ Stefano Cabrini \\ Lawrence Berkeley National Laboratory
}

See next page for additional authors

Follow this and additional works at: https://digitalcommons.unl.edu/physicsstreubel

Part of the Atomic, Molecular and Optical Physics Commons, Condensed Matter Physics Commons, and the Other Physics Commons

Wang, Szu Fan; Chorazewicz, Kayetan; Lamichhane, Suvechhya; Parrott, Ronald A.; Cabrini, Stefano; Fischer, Peter; Kent, Noah; Turner, John H.; Ishibashi, Takayuki; Frohock, Zachary Parker; Wisser, Jacob J.; Li, Peng; Zielinski, Ruthi; Herrington, Bryce; Suzuki, Yuri; Wu, Mingzhong; Munechika, Keiko; PinaHernandez, Carlos; Streubel, Robert; and Sweet, Allen A., "Ferromagnetic resonances in single-crystal yttrium iron garnet nanofilms fabricated by metal-organic decomposition" (2021). Robert Streubel Papers. 1.

https://digitalcommons.unl.edu/physicsstreubel/1

This Article is brought to you for free and open access by the Research Papers in Physics and Astronomy at DigitalCommons@University of Nebraska - Lincoln. It has been accepted for inclusion in Robert Streubel Papers by an authorized administrator of DigitalCommons@University of Nebraska - Lincoln. 


\section{Authors}

Szu Fan Wang, Kayetan Chorazewicz, Suvechhya Lamichhane, Ronald A. Parrott, Stefano Cabrini, Peter Fischer, Noah Kent, John H. Turner, Takayuki Ishibashi, Zachary Parker Frohock, Jacob J. Wisser, Peng Li, Ruthi Zielinski, Bryce Herrington, Yuri Suzuki, Mingzhong Wu, Keiko Munechika, Carlos Pina-Hernandez, Robert Streubel, and Allen A. Sweet 


\section{Ferromagnetic resonances in single-crystal yttrium iron garnet nanofilms fabricated by metal-organic decomposition}

Cite as: Appl. Phys. Lett. 119, 172405 (2021); https://doi.org/10.1063/5.0067122

Submitted: 13 August 2021 • Accepted: 13 October 2021 • Published Online: 26 October 2021

Szu-Fan (Paul) Wang, Kayetan Chorazewicz, Suvechhya Lamichhane, et al.
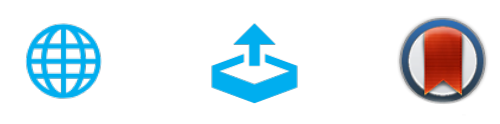

View Online

Export Citation

CrossMark

\section{ARTICLES YOU MAY BE INTERESTED IN}

Spin wave wavevector up-conversion in Y-shaped Permalloy structures

Applied Physics Letters 119, 172403 (2021); https://doi.org/10.1063/5.0068075

Substrate dependent reduction of Gilbert damping in annealed Heusler alloy thin films grown on group IV semiconductors

Applied Physics Letters 119, 172404 (2021); https://doi.org/10.1063/5.0060213

Breaking the symmetry of spin-sublattices in antiferromagnet by interfacial tailoring in the L1O-MnPt/ $\mathrm{NaCl} / \mathrm{Fe}$ junction

Applied Physics Letters 119, 172401 (2021); https://doi.org/10.1063/5.0064931

\section{Challenge us.}

What are your needs for periodic signal detection?

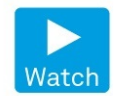

Zurich

- Instruments

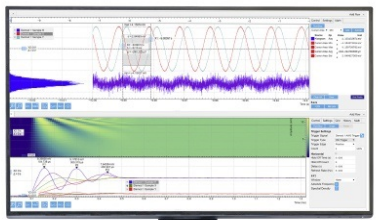

0 


\title{
Ferromagnetic resonances in single-crystal yttrium iron garnet nanofilms fabricated by metal-organic decomposition
}

Cite as: Appl. Phys. Lett. 119, 172405 (2021); doi: 10.1063/5.0067122

Submitted: 13 August 2021 - Accepted: 13 October 2021 •

Published Online: 26 October 2021

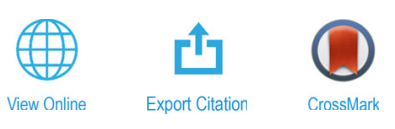

Szu-Fan (Paul) Wang, ${ }^{7}$ Kayetan Chorazewicz, ${ }^{2}$ Suvechhya Lamichhane, ${ }^{3}$ Ronald A. Parrott, ${ }^{1}$ iD Stefano Cabrini, ${ }^{4}$ Peter Fischer,, ${ }^{5,6}$ iD Noah Kent, ${ }^{5,6}$ John H. Turner, ${ }^{7}$ Takayuki Ishibashi, ${ }^{8}$ (D) Zachary Parker Frohock,

Jacob J. Wisser, ${ }^{10,11}$ (D) Peng Li, ${ }^{11,12}$ (D) Ruthi Zielinski, ${ }^{3}$ (iD Bryce Herrington, ${ }^{3}$ (D) Yuri Suzuki, ${ }^{10,11}$ Mingzhong Wu, ${ }^{9}$ Keiko Munechika, ${ }^{13}$ Carlos Pina-Hernandez, ${ }^{13}$ Robert Streubel, ${ }^{3,14, a)}$ (iD and Allen A. Sweet ${ }^{1,15, a)}$ (iD)

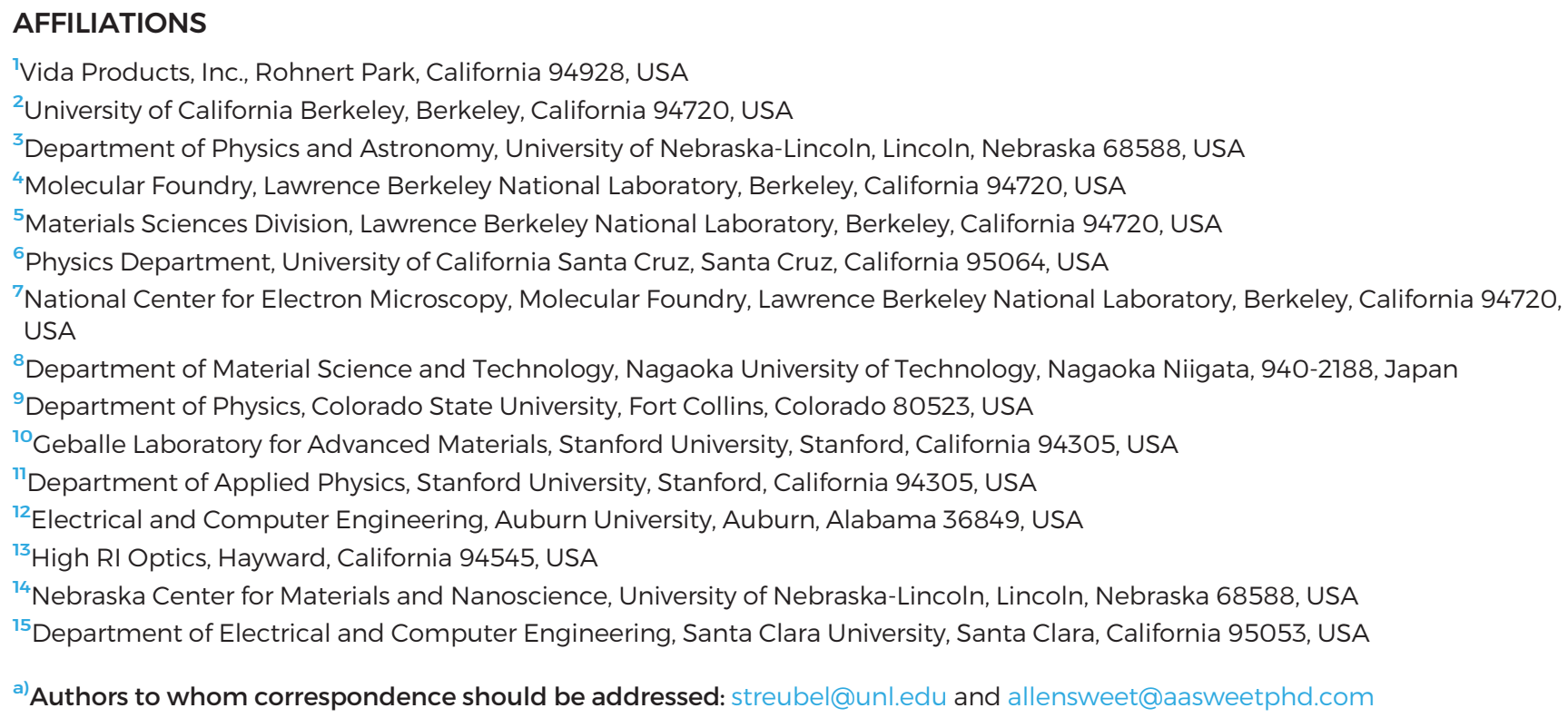

Tunable microwave and millimeter wave oscillators and bandpass filters with ultra-low phase noise play a critical role in electronic devices, including wireless communication, microelectronics, and quantum computing. Magnetic materials, such as yttrium iron garnet (YIG), possess ultra-low phase noise and a ferromagnetic resonance tunable up to tens of gigahertz. Here, we report structural and magnetic properties of single-crystal 60 and $130 \mathrm{~nm}$-thick YIG films prepared by metal-organic decomposition epitaxy. These films, consisting of multiple homoepitaxially grown monolayers, are atomically flat and possess magnetic properties similar to those grown with liquid-phase epitaxy, pulsed laser deposition, and sputtering. Our approach does not involve expensive high-vacuum deposition systems and is a true low-cost alternative to current commercial techniques that have the potential to transform the industry.
\end{abstract}

Published under an exclusive license by AIP Publishing. https://doi.org/10.1063/5.0067122

Tunable oscillators ${ }^{1}$ and bandpass filters ${ }^{2}$ are critical to the functionality of many electronic devices, including cellular communication, microelectronics, and quantum computing. Compared with traditional varactor diode-tuned oscillator technology, ${ }^{3}$ ferromagnetic resonance-based oscillators with tunable resonance and low damping promise a significant increase in the data rate of emerging $5 \mathrm{G}$ cellular networks ${ }^{4}$ owing to a reduced oscillator phase noise $(40 \mathrm{~dB})$ and the commercialization of magnonic electronics, ${ }^{5}$ transistors, ${ }^{6,7}$ and logic 
gates $^{8}$ and harnessing the inverse spin Hall effect; ${ }^{9-11}$ spin pumping; ${ }^{12-15}$ magnetostatic surface spin wave-based delay lines; ${ }^{16}$ and microwave isolators, circulators, and limiters. ${ }^{17}$ Wireless communication alone represents a market with roughly four billion people on earth owning a smart phone, an annual production of $1.5 \times 10^{9}$, and the world-wide rolling out of $5 \mathrm{G}$ cellular demanding ultra-low phase noise oscillators for high-data rate transmission. For these applications, yttrium iron garnet $\left(\mathrm{YIG}, \mathrm{Y}_{3} \mathrm{Fe}_{5} \mathrm{O}_{12}\right)^{18}$ and related garnets are the most likely candidates because of outstanding magnetic characteristics and the possibility of epitaxial growth of singlecrystal nanometer thick films or nanofilms, ${ }^{19}$ based on, e.g., liquidphase epitaxy (LPE), ${ }^{20}$ pulsed laser deposition (PLD), ${ }^{10,13,21-25}$ offaxis sputtering, ${ }^{26-31}$ and molecular beam epitaxy. ${ }^{32}$ These techniques involve high-vacuum fabrication tools, which are less suited for high-volume manufacturing of the kind needed for 5G cellular handsets and infrastructure. ${ }^{3}$

Here, we report structural and magnetic properties, including ferromagnetic resonances, of epitaxial YIG nanofilms fabricated by metal-organic decomposition $(\mathrm{MOD})^{34}$-an inexpensive alternative due to low process fabrication costs and low capital equipment costs. Epitaxial atomically flat layers with thicknesses up to $500 \mathrm{~nm}$ are grown through repeated application of MOD epitaxy. The saturation magnetization, gyromagnetic ratio, effective Gilbert damping, and inhomogeneous line broadening are very similar to and, in some cases, even better than those obtained by existing high-vacuum deposition techniques. The experimental data obtained from broadband spectroscopy are consistent with micromagnetic simulations and reveal a quality factor for in-plane geometry of 200-300 (experiment) and 2000 (simulation) at $20 \mathrm{GHz}$. The linear increase in the quality factor with excitation frequency observed in both experiment and simulations is essential to high-frequency applications.

The epitaxial YIG nanofilms are synthesized from a FeY-03(5/3) precursor solution (Kojundo Chemical Laboratory Co., Ltd.) containing 1.6 wt. $\% \mathrm{Fe}_{2} \mathrm{O}_{3}$ and 1.4 wt. $\% \mathrm{Y}_{2} \mathrm{O}_{3}$. The solution is spin-coated at $500 \mathrm{rpm}$ for $10 \mathrm{~s}$ followed by $2000 \mathrm{rpm}$ for $20 \mathrm{~s}$ onto $(5 \times 5) \mathrm{mm}^{2}$ and $(10 \times 10) \mathrm{mm}^{2}$ gadolinium gallium garnet(111) $\left(\mathrm{GGG}, \mathrm{Gd}_{3} \mathrm{Ga}_{5} \mathrm{O}_{12}\right)$ substrates [MTI Corporation and University Wafer (UW)] and dried for $24 \mathrm{~h}$ at room temperature to evaporate organic volatiles. The drying procedure can be accelerated to $1 \mathrm{~h}$ if heated to $150^{\circ} \mathrm{C}$. The dried compound is heated to $1100^{\circ} \mathrm{C}$ for $4 \mathrm{~h}$ in a quartz tub furnace in the presence of a research-grade oxygen atmosphere. During this annealing process, the layer crystallizes in a three-step process: decomposition of the metal-organic compounds (pyrolysis); elimination of the remaining organic material through annealing in oxygen atmosphere; and migration of metal atoms to form the YIG lattice structure according to the GGG substrate (lattice constants: $a_{\mathrm{YIG}}=12.38 \AA$; $a_{\mathrm{GGG}}$ $=12.37 \AA$ ). The resulting heteroepitaxial layer is atomically flat with a surface roughness $\leqq 0.2 \mathrm{~nm}$ for MTI GGG substrates [Fig. 1(a)], $(60 \pm 20) \mathrm{nm}$ thick, and according to electron backscatter diffraction single-crystalline [Fig. 1(b)]. Spin-coating and annealing on a non-lattice-matched substrate, such as silicon, yield a polycrystalline structure [Fig. 1(b)]. The absolute orientation of the crystallographic axis of the single-crystal YIG nanofilms is determined from x-ray diffraction [Fig. 1(c)]. Remarkably, repetitive spin coating and annealing to synthesize bilayers and multilayers show no signs of boundary layer discontinuity or increased surface roughness corroborating homoepitaxial growth for up to at least ten layers. This threshold is the result of our studies limited to a maximum of ten repetitions. Monolayer and

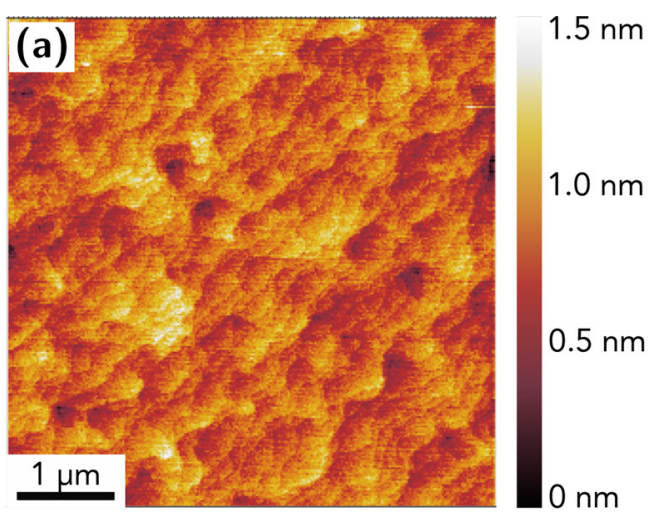

(b)
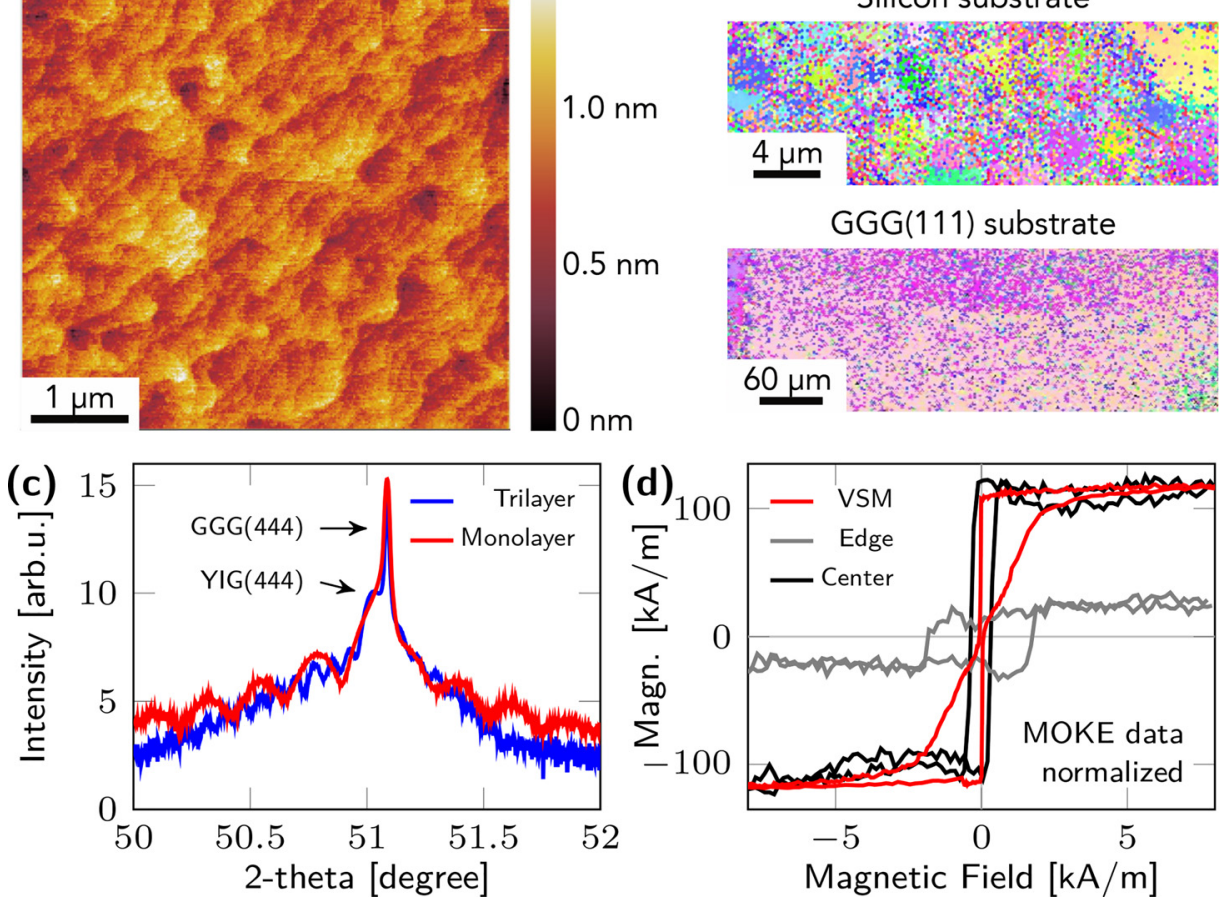

FIG. 1. Structural and magnetic properties of YIG nanofilms synthesized using metalorganic decomposition epitaxy. (a) Atomic force microscopy revealing atomic terrace formation. (b) Electron backscatter diffraction of YIG nanofilms prepared on (left) Si and (right) GGG(111) demonstrating polycrystalline and single-crystalline epitaxial growth. (c) X-ray diffraction of monolayer and trilayer films corroborating singlecrystallinity and homoepitaxy. (d) In-plane magnetic hysteresis loop obtained for $(2.5 \times 2.5) \mathrm{mm}^{2}$ corner pieces at room temperature from vibrating sample magnetometry and magneto-optical Kerr effect magnetometry (scaled) revealing the spatial variations in the coercivity and saturation magnetization. 
trilayer films, investigated in detail below, are 57 and $130 \mathrm{~nm}$ thick, respectively, according to x-ray diffraction [Fig. 1(c)]. A constant surface roughness $\leq 0.2 \mathrm{~nm}$ [Fig. 1(a)] is assigned to the atomic terrace formation.

These structural properties translate to a coercive field of (80-400) $\mathrm{A} / \mathrm{m}$ and a saturation magnetization ranging from 120 to $140 \mathrm{kA} / \mathrm{m}$ [Fig. 1(d); Table I], which is in good agreement with the bulk single-crystal values of $140 \mathrm{kA} / \mathrm{m}$. A closer look at the magnetic hysteresis loops obtained at room temperature with vibrating sample magnetometry (VSM, Quantum Design DynaCool PPMS) and magneto-optical Kerr effect magnetometry (MOKE) reveals local variations in the magnetic properties due to spin coating-induced thicker edge regions ( $\$ 1 \mathrm{~mm}$-wide) with impaired crystallization yielding the larger coercive field and smaller saturation magnetization. The optical setup features a $30 \mathrm{~mW} 639 \mathrm{~nm}$ continuous wave diode laser, whose intensity is modulated at $2 \mathrm{kHz}$ with a mechanical chopper that is synchronized with a $\mathrm{kHz}$ lock-in amplifier and provides a spatial resolution of about $10 \mu \mathrm{m}$. An initial annealing of the GGG substrate in a research-grade oxygen atmosphere at $1100^{\circ} \mathrm{C}$ for $4 \mathrm{~h}$ promotes (111) surface reconstruction, minimizes roughness [(1.0-2.5) $\AA$ ], and, in turn, reduces strain and magnetic anisotropy. The better crystal quality of MTI GGG compared with UW GGG results in smoother epitaxial YIG nanofilms and overall better structural and magnetic properties (Table I).

The ferromagnetic resonances in the YIG nanofilms are studied using broadband spectroscopy at room temperature and ambient conditions. The $(5 \times 5) \mathrm{mm}^{2}$ sample is centered and placed face-down on a coplanar waveguide with a conductor width of $250 \mu \mathrm{m}$, which avoids contributions from edge/corner regions with distinct magnetic properties [Fig. 1(d)]. The field derivative of the ferromagnetic resonance absorption intensity is acquired using a radio frequency diode combined with a $700 \mathrm{~Hz}$ modulation field while applying an ac excitation magnetic field at a constant frequency and sweeping the dc magnetic field across the resonance [Fig. 2(a)]. Each spectrum is fitted with the derivative of the sum of symmetric and asymmetric Lorentzians to extract resonance field, intensity, and full width at half maximum (FWHM) $\Delta H(f)$. No secondary standing spin-wave mode is observed in the epitaxial single-crystalline films. The dispersion $f(H)$ is assembled for in-plane and out-of-plane geometries to quantify the effective Gilbert damping constant $\alpha$, inhomogeneous line broadening $\Delta H(0)$, and absolute gyromagnetic ratio $\gamma=g \mu_{B} / \hbar$ with the Bohr magneton $\mu_{B}$ and Landé factor $g$ (Table I). For in-plane measurements, the resonance frequency $f_{\text {res }}$ relates to the resonance field $H_{\text {res }}$ via $2 \pi f_{\text {res }}^{\|}$ $\left.=\gamma \mu_{0} \sqrt{\left(H_{r e s}^{\|}+H_{i p}\right)\left(H_{r e s}^{\|}+H_{i p}+H_{k}+M_{s}\right)}\right)^{36,37}$ Using the saturation magnetization from vibrating sample magnetometry, the fits corroborate negligible in-plane $\left(H_{i p}\right)$ and perpendicular $\left(H_{k}\right)$ magnetic anisotropy fields $<2 \mathrm{kA} / \mathrm{m}$, and a gyromagnetic ratio $\gamma \approx 0.22 \mathrm{MHz} /$ $(\mathrm{A} / \mathrm{m})$ that is nearly independent of film thicknesses and substrates and similar to the free-electron values of bulk YIG material, PLD, and LPE [Fig. 2(b); Table I]. A similar picture is drawn by out-of-plane measurements, where, for a negligible anisotropy, the dispersion reads $2 \pi f_{\text {res }}^{\perp}=\gamma \mu_{0}\left(H_{\text {res }}^{\perp}-M_{\text {eff }}\right){ }^{36,37}$ The resonance frequency is significantly smaller due to demagnetization field contributions represented by the effective saturation magnetization (in the normal direction) $M_{e f f}=M_{s}-H_{k} \approx M_{s}$.

Plotting the FWHM resonance linewidth $\Delta H(f)=\Delta H(0)$ $+4 \pi \alpha_{\text {int }} f / \gamma+\Delta H_{\Gamma}$ reveals, for in-plane geometry, a strong nonlinearity with the excitation frequency [Fig. 2(c)]. The effective Gilbert damping $\alpha$ given in Table I is retrieved from linear fitting the linewidth in the range $(5-10) \mathrm{GHz}$ without considering $\Delta H_{\Gamma}$, which would yield a significantly lower intrinsic value $\alpha_{i n t}$ and prevent a comparison with the literature. Furthermore, the effective damping is essential for applications. We focus on the in-plane geometry because of its relevance to high-frequency applications and, in our case, a less reliable linewidth for out-of-plane geometry measurements. The term $\Delta H_{\Gamma}$

TABLE I. Magnetic properties of epitaxial YIG nanofilms prepared by metal-organic decomposition compared with liquid-phase epitaxy (LPE), pulsed laser deposition (PLD), and sputtering. Saturation magnetization $M_{S}$ is obtained from vibrating sample magnetometry. Gyromagnetic ratio $\gamma$, inhomogeneous line broadening $\Delta H(0)$, and effective Gilbert damping $\alpha$ for $(5-10) \mathrm{GHz}$ are retrieved from broadband ferromagnetic resonance spectroscopy in in-plane and out-of-plane geometries probing a $250 \mu \mathrm{m}$-wide region of $(5 \times 5) \mathrm{mm}^{2}$ samples. Film thickness: M1, M2, U1: $(60 \pm 20) \mathrm{nm}$; M3, M4, U3: $(130 \pm 20) \mathrm{nm}$. All samples but U3 are annealed.

\begin{tabular}{|c|c|c|c|c|}
\hline Sample & $M_{s}(\mathrm{kA} / \mathrm{m})$ & $\gamma[\mathrm{MHz} /(\mathrm{A} / \mathrm{m})]$ & $\Delta H(0)(\mathrm{kA} / \mathrm{m})$ & $\alpha^{1}$ \\
\hline \multicolumn{5}{|c|}{ In-plane; YIG on MTI GGG(111) } \\
\hline M1 & $130.3 \pm 8.9$ & $0.22(1)$ & $0.23(1)$ & $0.0002(2)$ \\
\hline M2 & $122.3 \pm 6.9$ & $0.22(1)$ & $0.45(1)$ & $0.0004(2)$ \\
\hline M3 & $139.9 \pm 8.9$ & $0.22(1)$ & $0.45(1)$ & $0.0002(2)$ \\
\hline M4 & $139.0 \pm 11.6$ & $0.22(1)$ & $0.43(2)$ & $0.0003(2)$ \\
\hline \multicolumn{5}{|c|}{ In-plane; YIG on UW GGG(111) } \\
\hline $\mathrm{U} 1$ & $133.7 \pm 8.0$ & $0.22(1)$ & $1.37(5)$ & $0.0004(3)$ \\
\hline U3 & $130.1 \pm 10.0$ & $0.22(1)$ & $2.32(5)$ & $0.0025(5)$ \\
\hline \multicolumn{5}{|c|}{ Out-of-plane; YIG on UW GGG(111) } \\
\hline U1 & $133.7 \pm 8.0$ & $0.22(1)$ & $0.15(5)$ & $0.0023(10)$ \\
\hline \multicolumn{5}{|c|}{ Literature values } \\
\hline $\mathrm{LPE}^{20}$ & $131.6-147.6$ & 0.22 & $0.11-0.16$ & $>0.0004$ \\
\hline $\operatorname{PLD}^{10,21,24}$ & 137.2 & 0.22 & $0.10-0.27$ & $>0.0003$ \\
\hline Sputt. ${ }^{28,31,35}$ & $130.8-142.0$ & 0.22 & $0.55-1.99$ & $>0.0001$ \\
\hline
\end{tabular}



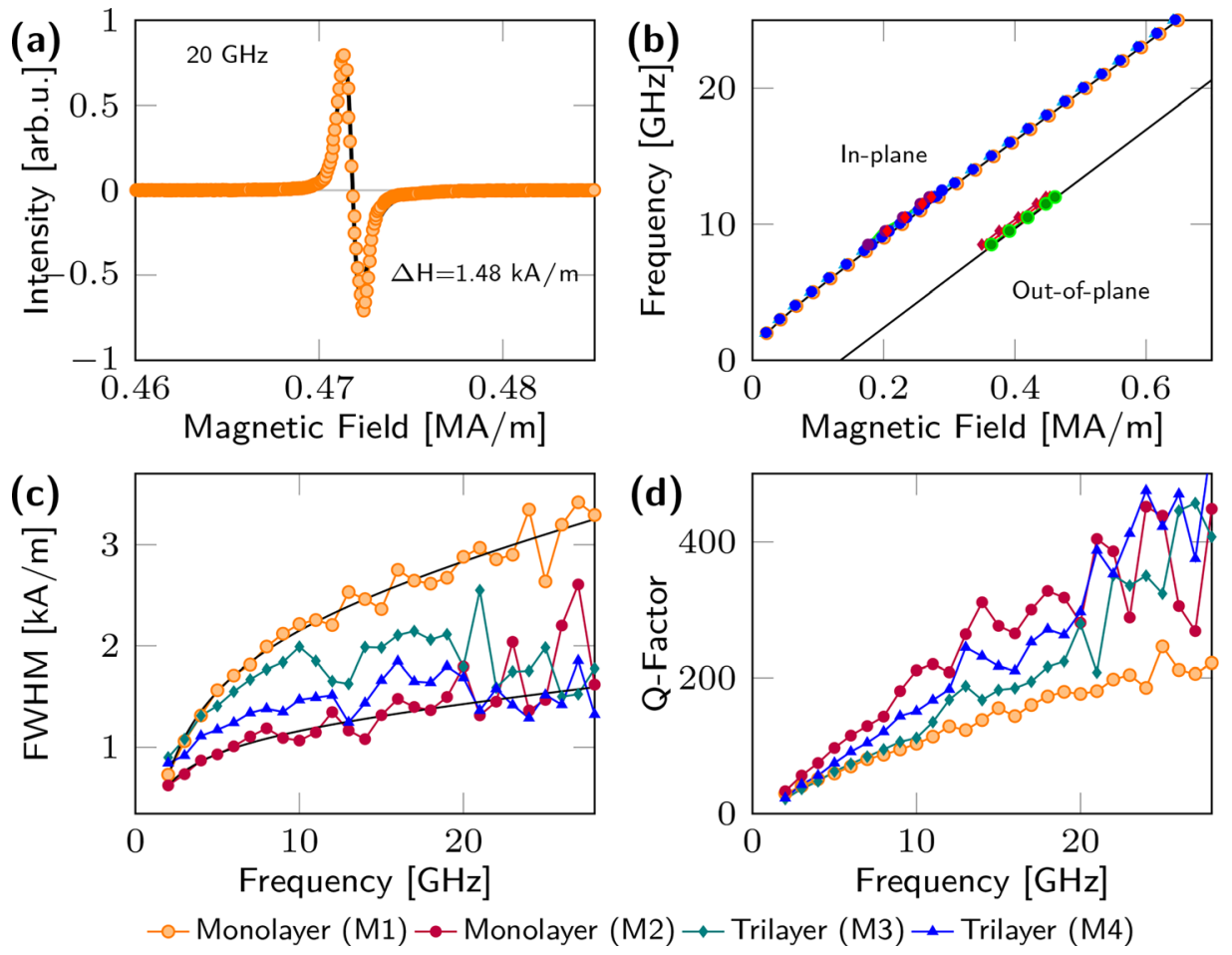

FIG. 2. Experimental ferromagnetic resonance spectroscopy probing a $250 \mu \mathrm{m}$-wide central region of $(5 \times 5) \mathrm{mm}^{2}$ samples at room temperature. (a) Intensity across ferromagnetic resonance at $20 \mathrm{GHz}$ and in the in-plane geometry. (b) Dispersion for various epitaxial YIG nanofilms revealing nearly the same frequency independent of film thicknesses and substrates. The dispersion for in-plane and out-of-plane geometries is fitted using the Kittel equations discussed in the text. (c) Full width at half maximum for various YIG nanofilms demonstrating significant contributions from two-magnon scattering (fit). (d) Quality factor linearly increasing with resonance frequency. (c) and (d) are shown for in-plane geometry and refer to the same data.

describes the line broadening of a nearly uniform precession mode in epitaxial films due to two-magnon scattering ${ }^{38-42}$ on defects $\Delta H_{\Gamma}=\Gamma \sin ^{-1} \sqrt{\frac{\sqrt{f^{2}+f_{M}^{2}}-f_{M}^{2}}{\sqrt{f^{2}+f_{M}^{2}}+f_{M}^{2}}}$ with $f_{M}=\gamma \mu_{0} M_{s} / 2 \pi$. This classical twomagnon model for strong exchange ${ }^{43}$ and dipole ${ }^{44}$ interactions, without considering modifications to the spin-wave density of states, ${ }^{45-47}$ is valid for small low-density defects and describes the eigenmode mixing. ${ }^{43}$ Specifically, point defects, nanoscale clusters, strain, and dislocations may cause uniformly precessing magnetic moments to dephase local magnon modes with characteristic frequencies because of translational symmetry breaking of the magnetic system through, e.g., localized dipolar fields (variations in the saturation magnetization). ${ }^{43,48,49}$ The two-magnon scattering contribution, quantified by $\Gamma,{ }^{43,48,49}$ varies between 0.9 and $1.8 \mathrm{kA} / \mathrm{m}$ due to varying YIG epitaxy. Among the many nominally same samples, only M3 and M4 reveal similar dynamic magnetic properties, illustrating the need for future processes and film optimization and the higher sensitivity of magnon scattering compared with $\mathrm{x}$-ray diffraction to local defects existing at internal interfaces and in bulk. Nonetheless, the quality factor $Q=H_{\text {res }} / \Delta H$ linearly increases with the frequency in the entire frequency range and for all samples [Fig. 2(d)].

To augment these experimental data and explore the maximal quality factor possible at a reasonable resonance intensity, we perform micromagnetic simulations using Boris computational spintronics ${ }^{50}$ and the Landau-Lifshitz-Gilbert formalism at $0 \mathrm{~K}$. The YIG nanofilm is modeled as a rectangular film $\left(250 \times 250 \mu \mathrm{m}^{2} \times t\right)$ with periodic boundary conditions along $x$ and $y$ axes and film thicknesses $t=10,60$, and $130 \mathrm{~nm}$. The mesh discretization along all three directions is half of the magneto-static exchange length, i.e., $\frac{1}{2} \sqrt{A /\left(\frac{1}{2} \mu_{0} M_{s}^{2}\right)}=8.7 \mathrm{~nm}$. The discretization in the normal direction of $10 \mathrm{~nm}$-thick films is $5 \mathrm{~nm}$. These values are ten times smaller than the magneto-crystalline exchange length $\sqrt{A /|K|}=78 \mathrm{~nm}$. This estimation and numerical modeling are based on the following YIG material parameters: Heisenberg exchange $J=3.7 \mathrm{pJ} / \mathrm{m},{ }^{51}$ perpendicular magnetic anisotropy $K=-600 \mathrm{~J} / \mathrm{m}^{3} \quad$ (in-plane easy-plane), ${ }^{25,52}$ and $M_{s}=140 \mathrm{kA} / \mathrm{m}$, $\alpha=0.0005$, and $g=2[\gamma=0.22 \mathrm{MHz} /(\mathrm{A} / \mathrm{m})]$ chosen according to experimental data (Table I). The demagnetization field is calculated using multilayered convolution. The magnetization is excited via a magnetic field pulse $\left[\operatorname{sinc}\left\{2 \pi f_{c} \cdot\left(t-t_{f} / 2\right)\right\} \mathrm{kA} / \mathrm{m}\right]$ along $x$ and perpendicular to the dc magnetic field ( $y$ for in-plane and $z$ for out-of-plane). To provide sufficient temporal and frequency resolution, a simulation time of $t_{f}=100 \mathrm{~ns}$ and a cutoff frequency of $f_{c}=400 \mathrm{GHz}$ are used. The latter enables us to probe magnon modes up to this value. The dc magnetic field is varied from $50 \mathrm{kA} / \mathrm{m}$ to $1 \mathrm{MA} / \mathrm{m}$ in steps of $25 \mathrm{kA} / \mathrm{m}$. For all cases, the dispersion is linear, possesses the same slope, and shows excellent agreement with the experimental data (Fig. 3). Resonances in the out-of-plane geometry reveal a film thickness dependence that coincides with the $60 \mathrm{~nm}$-thick films [Fig. 3(b)], and the in-plane spectra of $10 \mathrm{~nm}$-thick films unveil a sizable satellite peak at $f_{\text {res }}^{\|}-0.7 \mathrm{GHz}$. Similar to the experimental data, the modeled quality factors $Q=$ $f_{\text {res }} / \Delta f$ for the in-plane geometry increases with the resonance frequency and exceeds 1000 at $20 \mathrm{GHz}$. The latter is ten times larger than the experimental values likely due to the omission of disorder and twomagnon scattering. The large, yet systematic, variations in the quality factor are due to limited resolution in the reciprocal space despite the $100 \mathrm{~ns}$ simulation time. For the out-of-plane geometry, a saturation value of 1000 is observed. This difference between in-plane and out-of- 

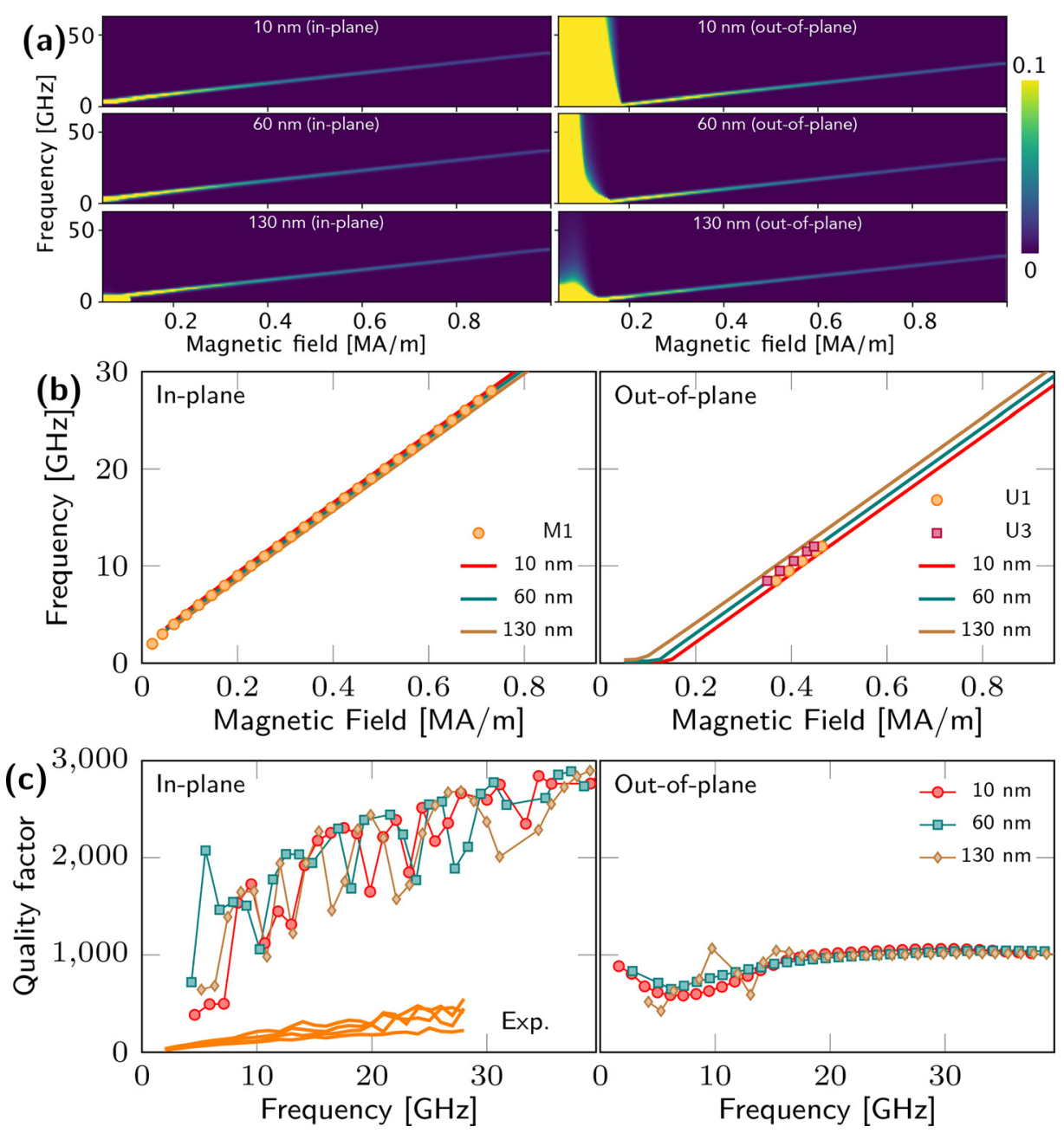

FIG. 3. Numerical modeling of ferromagnetic resonances in YIG nanofilms. (a) Intensity maps for in-plane and out-ofplane geometries showing a linear increase in the resonance frequency with field and relatively small changes in line broadening. (b) Dispersion revealing thickness dependence of the out-of-plane geometry and virtually no change for the in-plane geometry. Experimental data are added for comparison. (c) Quality factor for in-plane and out-of-plane geometries showcasing a linear increase with frequency and constant values, respectively. plane geometries is consistent with the experimental Gilbert damping values, which are indirectly proportional to the quality factor.

In conclusion, the epitaxial YIG nanofilms prepared by metalorganic decomposition possess similar magnetic properties as those synthesized by high-vacuum techniques that can be analytically and numerically described. The resonance frequency is virtually independent of the film thickness and substrates. Variations in the film quality are apparent by a sizable indeterministic effect on resonance linewidth, effective Gilbert damping, and quality factor due to two-magnon scattering. The in-plane quality factor $(200-300$ at $20 \mathrm{GHz})$ increases linearly with excitation frequency and sets ferromagnetic resonancebased oscillators apart from traditional varactor diode-tuned oscillators $[Q<2$ at the $\mathrm{C}$-band $5 \mathrm{G}$ frequencies $(\sim 3.5 \mathrm{GHz})]$. Numerical modeling of YIG nanofilms without structural defects predicts a quality factor $>1000$. This increase, obtained with an economical synthesis process, is significant for high-frequency $5 \mathrm{G}$ applications. A fully functional YIG oscillator or filter will require a magnetic field to tune the ferromagnetic resonance or, as shown by simulations, to tailor the magnetic anisotropy of the YIG nanofilm. While the latter is not feasible, the former can be achieved using an electromagnet (currentdriven selection of the resonance frequency) and/or a permanent magnet (energy efficiency).

We would like to thank Caroline A. Ross (Massachusetts Institute of Technology) for fruitful discussions on polycrystalline YIG films grown on silicon. The work at the Molecular Foundry was supported by the U.S. Department of Energy, Office of Science, Basic Energy Sciences under Contract No. DE-AC02-05-CH11231. This work was supported by the Jane Robertson Layman Fund held at the University of Nebraska Foundation and Nebraska EPSCoR under the FIRST Award No. OIA-1557417. P.F. and N.K. acknowledge support by the U.S. Department of Energy, Office of Science, Office of Basic Energy Sciences, Materials Sciences and Engineering Division under Contract No. DE-AC02-05-CH11231 (NEMM program MSMAG). Z.P.F. and M.W. acknowledge support by the U.S. National Science Foundation (Nos. EFMA1641989 and ECCS-1915849). The work at Stanford was funded by the Vannevar Bush Faculty Fellowship program sponsored by the 
Basic Research Office of the Assistant Secretary of Defense for Research and Engineering and funded by the Office of Naval Research through Grant No. N00014-15-1-0045 (P.L.) and the U.S. Department of Energy, Director, Office of Science, Office of Basic Energy Sciences, Division of Materials Sciences and Engineering under Contract No. DESC0008505 (J.J.W. and Y.S.).

\section{AUTHOR DECLARATIONS \\ Conflict of Interest}

The authors have no conflicts to disclose.

\section{DATA AVAILABILITY}

The data that support the findings of this study are available from the corresponding authors upon reasonable request.

\section{REFERENCES}

${ }^{1}$ A. A. Sweet and R. Parrott, "A wide band, low phase noise, differential YIG tuned oscillator," in WAMICON 2014 (Institute of Electrical and Electronics Engineers, 2014), pp. 1-3.

${ }^{2}$ C. Tsai, G. Qiu, H. Gao, L. Yang, G. Li, S. Nikitov, and Y. Gulyaev, “Tunable wideband microwave band-stop and band-pass filters using YIG/GGG-GaAs layer structures," IEEE Trans. Magn. 41, 3568-3570 (2005).

${ }^{3}$ A. A. Sweet, MIC and MMIC Amplifier and Oscillator Circuit Design (Artech House, 1990).

${ }^{4}$ Ericsson ConsumerLabFive Ways to a Better 5G: Key Trends Influencing Consumer Adoption of $5 G$ (Ericsson Research Report, 2019).

${ }^{5}$ Y. Kajiwara, K. Harii, S. Takahashi, J. Ohe, K. Uchida, M. Mizuguchi, H. Umezawa, H. Kawai, K. Ando, K. Takanashi, S. Maekawa, and E. Saitoh, "Transmission of electrical signals by spin-wave interconversion in a magnetic insulator," Nature 464, 262-266 (2010).

${ }^{6}$ A. V. Chumak, A. A. Serga, and B. Hillebrands, "Magnon transistor for allmagnon data processing," Nat. Commun. 5, 4700 (2014).

${ }^{7}$ A. Chumak, A. Serga, and B. Hillebrands, "Magnonic crystals for data processing," J. Phys. D: Appl. Phys. 50, 244001 (2017).

${ }^{8}$ A. Khitun, M. Bao, and K. L. Wang, "Magnonic logic circuits," J. Phys. D: Appl. Phys. 43, 264005 (2010).

${ }^{9}$ K. Ando, S. Takahashi, J. Ieda, Y. Kajiwara, H. Nakayama, T. Yoshino, K. Harii, Y. Fujikawa, M. Matsuo, S. Maekawa, and E. Saitoh, "Inverse spin-Hall effect induced by spin pumping in metallic system,” J. Appl. Phys. 109, 103913 (2011).

${ }^{10} \mathrm{O}$. d'Allivy Kelly, A. Anane, R. Bernard, J. Ben Youssef, C. Hahn, A. H. Molpeceres, C. Carrétéro, E. Jacquet, C. Deranlot, P. Bortolotti, R. Lebourgeois, J.-C. Mage, G. de Loubens, O. Klein, V. Cros, and A. Fert, "Inverse spin Hall effect in nanometer-thick yttrium iron garnet/Pt system," Appl. Phys. Lett. 103, 082408 (2013).

${ }^{11}$ H. Nakayama, M. Althammer, Y.-T. Chen, K. Uchida, Y. Kajiwara, D. Kikuchi, T. Ohtani, S. Geprägs, M. Opel, S. Takahashi, R. Gross, G. E. W. Bauer, S. T. B. Goennenwein, and E. Saitoh, "Spin Hall magnetoresistance induced by a nonequilibrium proximity effect,” Phys. Rev. Lett. 110, 206601 (2013).

${ }^{12}$ C. W. Sandweg, Y. Kajiwara, A. V. Chumak, A. A. Serga, V. I. Vasyuchka, M. B. Jungfleisch, E. Saitoh, and B. Hillebrands, "Spin pumping by parametrically excited exchange magnons," Phys. Rev. Lett. 106, 216601 (2011).

${ }^{13}$ B. Heinrich, C. Burrowes, E. Montoya, B. Kardasz, E. Girt, Y.-Y. Song, Y. Sun, and $\mathrm{M}$. Wu, "Spin pumping at the magnetic insulator (YIG)/normal metal (Au) interfaces," Phys. Rev. Lett. 107, 066604 (2011).

${ }^{14}$ C. Burrowes, B. Heinrich, B. Kardasz, E. A. Montoya, E. Girt, Y. Sun, Y.-Y. Song, and $\mathrm{M}$. Wu, "Enhanced spin pumping at yttrium iron garnet/Au interfaces," Appl. Phys. Lett. 100, 092403 (2012).

${ }^{15}$ S. M. Rezende, R. L. Rodríguez-Suárez, M. M. Soares, L. H. Vilela-Leão, D. Ley Domínguez, and A. Azevedo, "Enhanced spin pumping damping in yttrium iron garnet/Pt bilayers,” Appl. Phys. Lett. 102, 012402 (2013).

${ }^{16} \mathrm{M}$. A. Tsankov, M. Chen, and C. E. Patton, "Magnetostatic wave dynamic magnetization response in yttrium iron garnet films," J. Appl. Phys. 79, 1595-1603 (1996).
${ }^{17}$ M. Jamali, A. K. Smith, H. Li, and J.-P. Wang, "Evaluation of spin waves and ferromagnetic resonance contribution to the spin pumping in a $\mathrm{Ta} / \mathrm{CoFeB}$ structure," J. Phys. D: Appl. Phys. 49, 12LT01 (2016).

${ }^{18}$ G. Schmidt, C. Hauser, P. Trempler, M. Paleschke, and E. T. Papaioannou, "Ultra thin films of yttrium iron garnet with very low damping: A review," Phys. Status Solidi B 257, 1900644 (2020).

${ }^{19}$ J. Palisaitis, R. Vasiliauskas, and G. Ferro, "Epitaxial growth of thin films," in Physics of Advanced Materials Winter School (InTech, 2008), pp. 1-16.

${ }^{20}$ M. C. Onbasli, A. Kehlberger, D. H. Kim, G. Jakob, M. Kläui, A. V. Chumak, B. Hillebrands, and C. A. Ross, "Pulsed laser deposition of epitaxial yttrium iron garnet films with low Gilbert damping and bulk-like magnetization," APL Mater. 2, 106102 (2014).

${ }^{21}$ Y. Sun, Y.-Y. Song, H. Chang, M. Kabatek, M. Jantz, W. Schneider, M. Wu, H. Schultheiss, and A. Hoffmann, "Growth and ferromagnetic resonance properties of nanometer-thick yttrium iron garnet films," Appl. Phys. Lett. 101, 152405 (2012).

${ }^{22}$ C. Hauser, T. Richter, N. Homonnay, C. Eisenschmidt, M. Qaid, H. Deniz, D. Hesse, M. Sawicki, S. G. Ebbinghaus, and G. Schmidt, "Yttrium iron garnet thin films with very low damping obtained by recrystallization of amorphous material," Sci. Rep. 6, 20827 (2016).

${ }^{23}$ C. Tang, M. Aldosary, Z. Jiang, H. Chang, B. Madon, K. Chan, M. Wu, J. E. Garay, and J. Shi, "Exquisite growth control and magnetic properties of yttrium iron garnet thin films," Appl. Phys. Lett. 108, 102403 (2016).

${ }^{24}$ M. Balinskiy, S. Ojha, H. Chiang, M. Ranjbar, C. A. Ross, and A. Khitun, "Spin wave excitation in sub-micrometer thick $\mathrm{Y}_{3} \mathrm{Fe}_{5} \mathrm{O}_{12}$ films fabricated by pulsed laser deposition on garnet and silicon substrates: A comparative study," J. Appl. Phys. 122, 123904 (2017).

${ }^{25}$ J. Mendil, M. Trassin, Q. Bu, J. Schaab, M. Baumgartner, C. Murer, P. T. Dao, J. Vijayakumar, D. Bracher, C. Bouillet, C. A. F. Vaz, M. Fiebig, and P. Gambardella, "Magnetic properties and domain structure of ultrathin yttrium iron garnet/pt bilayers," Phys. Rev. Mater. 3, 034403 (2019).

${ }^{26}$ H. L. Glass, "Growth of thick single-crystal layers of yttrium iron garnet by liquid phase epitaxy,” J. Cryst. Growth 33, 183 (1976).

${ }^{27}$ H. L. Wang, C. H. Du, Y. Pu, R. Adur, P. C. Hammel, and F. Y. Yang, "Large spin pumping from epitaxial $\mathrm{Y}_{3} \mathrm{Fe}_{5} \mathrm{O}_{12}$ thin films to Pt and W layers," Phys. Rev. B 88, 100406 (2013).

${ }^{28}$ T. Liu, H. Chang, V. Vlaminck, Y. Sun, M. Kabatek, A. Hoffmann, L. Deng, and $\mathrm{M}$. Wu, "Ferromagnetic resonance of sputtered yttrium iron garnet nanometer films," J. Appl. Phys. 115, 17A501 (2014).

${ }^{29}$ H. L. Wang, C. H. Du, Y. Pu, R. Adur, P. C. Hammel, and F. Y. Yang, "Scaling of spin Hall angle in $3 \mathrm{~d}, 4 \mathrm{~d}$, and $5 \mathrm{~d}$ metals from $\mathrm{Y}_{3} \mathrm{Fe}_{5} \mathrm{O}_{12} /$ metal spin pumping," Phys. Rev. Lett. 112, 197201 (2014).

${ }^{30}$ H. Chang, P. Li, W. Zhang, T. Liu, A. Hoffmann, L. Deng, and M. Wu, "Nanometer-thick yttrium iron garnet films with extremely low damping," IEEE Magn. Lett. 5, 1 (2014).

${ }^{31}$ C. L. Jermain, S. V. Aradhya, N. D. Reynolds, R. A. Buhrman, J. T. Brangham, M. R. Page, P. C. Hammel, F. Y. Yang, and D. C. Ralph, "Increased lowtemperature damping in yttrium iron garnet thin films," Phys. Rev. B 95, 174411 (2017).

${ }^{32}$ C. L. Jermain, H. Paik, S. V. Aradhya, R. A. Buhrman, D. G. Schlom, and D. C. Ralph, "Low-damping sub-10-nm thin films of lutetium iron garnet grown by molecular-beam epitaxy," Appl. Phys. Lett. 109, 192408 (2016).

${ }^{33}$ R. Carter, J. Owens, and D. De, "YIG oscillators: Is a planar geometry better?," IEEE Trans. Microwave Theory Tech. 32, 1671 (1984).

${ }^{34}$ T. Ishibashi, T. Kawata, T. H. Johansen, J. He, N. Harada, and K. Sato, "Magneto-optical indicator garnet films grown by metal-organic decomposition method," J. Magn. Soc. Jpn. 32, 150-153 (2008).

${ }^{35} \mathrm{C}$. Chang and A. Sakdinawat, "Ultra-high aspect ratio high-resolution nanofabrication for hard x-ray diffractive optics," Nat. Commun. 5, 4243 (2014).

${ }^{36} \mathrm{C}$. Kittel, "On the theory of ferromagnetic resonance absorption," Phys. Rev. 73, 155-161 (1948).

${ }^{37}$ M. Farle, "Ferromagnetic resonance of ultrathin metallic layers," Rep. Prog. Phys. 61, 755 (1998).

${ }^{38}$ J. Lindner, K. Lenz, E. Kosubek, K. Baberschke, D. Spoddig, R. Meckenstock, J. Pelzl, Z. Frait, and D. L. Mills, "Non-gilbert-type damping of the magnetic relaxation in ultrathin ferromagnets: Importance of magnon-magnon scattering," Phys. Rev. B 68, 060102 (2003). 
${ }^{39} \mathrm{G}$. Woltersdorf and B. Heinrich, "Two-magnon scattering in a self-assembled nanoscale network of misfit dislocations," Phys. Rev. B 69, 184417 (2004).

${ }^{40} \mathrm{R}$. McMichael and P. Krivosik, "Classical model of extrinsic ferromagnetic resonance linewidth in ultrathin films," IEEE Trans. Magn. 40, 2-11 (2004).

${ }^{41} \mathrm{~K}$. Lenz, H. Wende, W. Kuch, K. Baberschke, K. Nagy, and A. Jánossy, "Twomagnon scattering and viscous gilbert damping in ultrathin ferromagnets," Phys. Rev. B 73, 144424 (2006).

${ }^{42}$ S. Emori, U. S. Alaan, M. T. Gray, V. Sluka, Y. Chen, A. D. Kent, and Y. Suzuki, "Spin transport and dynamics in all-oxide perovskite $\mathrm{La}_{2 / 3} \mathrm{Sr}_{1 / 3} \mathrm{MnO}_{3} /$ $\mathrm{SrRuO}_{3}$ bilayers probed by ferromagnetic resonance," Phys. Rev. B 94, 224423 (2016).

${ }^{43}$ R. D. McMichael, D. J. Twisselmann, and A. Kunz, "Localized ferromagnetic resonance in inhomogeneous thin films," Phys. Rev. Lett. 90, 227601 (2003).

${ }^{44} \mathrm{~S}$. Geschwind and A. M. Clogston, "Narrowing effect of dipole forces on inhomogeneously broadened lines," Phys. Rev. 108, 49-53 (1957).

${ }^{45} \mathrm{Q}$. H. F. Vrehen, "Absorption and dispersion in porous and anisotropic polycrystalline ferrites at microwave frequencies," J. Appl. Prrhys. 40, 1849-1860 (1969).
${ }^{46}$ Q. H. F. Vrehen, A. B. van Groenou, and J. G. M. de Lau, "Relaxation of ferromagnetic precession by excitation of spin waves in polycrystalline nickel-cobalt ferrites," Phys. Rev. B 1, 2332-2347 (1970).

${ }^{47} \mathrm{E}$. Schlömann, "Inhomogeneous broadening of ferromagnetic resonance lines," Phys. Rev. 182, 632-645 (1969).

${ }^{48} \mathrm{R}$. Arias and D. L. Mills, "Extrinsic contributions to the ferromagnetic resonance response of ultrathin films," Phys. Rev. B 60, 7395-7409 (1999).

${ }^{49} \mathrm{R}$. Arias and D. L. Mills, "Extrinsic contributions to the ferromagnetic resonance response of ultrathin films," J. Appl. Phys. 87, 5455-5456 (2000).

${ }^{50} \mathrm{~S}$. Lepadatu, "Boris computational spintronics-High performance multi-mesh magnetic and spin transport modeling software," J. Appl. Phys. 128, 243902 (2020).

${ }^{51}$ S. Klingler, A. Chumak, T. Mewes, B. Khodadadi, C. Mewes, C. Dubs, O. Surzhenko, B. Hillebrands, and A. Conca, "Measurements of the exchange stiffness of YIG films using broadband ferromagnetic resonance techniques," J. Phys. D: Appl. Phys. 48, 015001 (2015).

${ }^{52} \mathrm{P}$. Hansen, "Anisotropy and magnetostriction of gallium-substituted yttrium iron garnet," J. Appl. Phys. 45, 3638-3642 (1974). 